Donald Cardwell

Viewegs Geschichte der Technik 
In Viewegs Reihe zur Geschichte der Naturwissenschaften wollen wir neue Aspekte und Interpretationen der Wissenschaftsgeschichte einem breiten Publikum zugänglich machen. International anerkannte Historiker schreiben allgemeinverständlich über ihr Spezialgebiet und zeigen so spannende Zusammenhänge der Wissenschaftsentwicklungen in den letzten Jahrhunderten auf.

Peter J. Bowler

Viewegs Geschichte der Umweltwissenschaften

William H. Brock

Viewegs Geschichte der Chemie

Donald Cardwell

Viewegs Geschichte der Technik

John North

Viewegs Geschichte der Astronomie und Kosmologie 
Donald Cardwell

\title{
Viewegs \\ Geschichte der Technik
}

\author{
Aus dem Englischen übersetzt \\ von Peter Hiltner
}

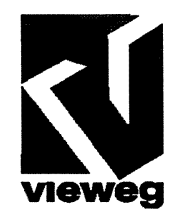


Titelgraphik:

Pläne für die Menai-Hängebrücke sowie die Eisenbrücke über den Fluß Severn von Thomas Telford

Originalausgabe:

(C) Fontana Press, Imprint of Harper Collins Publishers,

London 1994

Authorised translation from English language edition

"The fontana history of Technology".

Alle Rechte vorbehalten

(C) Friedr. Vieweg \& Sohn Verlagsgesellschaft mbH, Braunschweig/Wiesbaden, 1997

Softcover reprint of the hardcover 1st edition 1997

Der Verlag Vieweg ist ein Unternehmen der Bertelsmann Fachinformation GmbH.

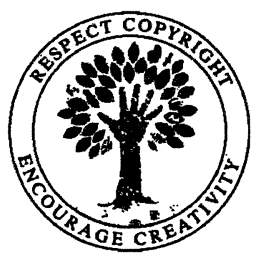

Das Werk einschließlich aller seiner Teile ist urheberrechtlich geschützt. Jede Verwertung außerhalb der engen Grenzen des Urheberrechtsgesetzes ist ohne Zustimmung des Verlags unzulässig und strafbar. Das gilt insbesondere für Vervielfältigungen, Übersetzungen, Mikroverfilmungen und die Einspeicherung und Verarbeitung in elektronischen Systemen.

Gedruckt auf säurefreiem Papier 


\section{Vorwort}

Die Geschichte der Technik beginnt bereits mit dem sogenannten „eotechnischen“ oder frühtechnischen Zeitalter und den einfachsten Werkzeugen und Handwerkskünsten. Faktisch beginnt die Geschichte des Homo sapiens mit den ersten Werkzeugen, die von Archäologen gefunden wurden; sie setzt sich dann fort durch die verschiedensten Kulturen und Zivilisationen bis in die heutige Epoche der Computer und der Raumfahrt. Es handelt sich um die längste, die allgemeinste und, wie man sagen kann, die fundamentalste Form der weltlichen Geschichtsschreibung. Sie ist am wenigsten mit lokalen, nationalen oder rassistischen Vorurteilen belastet - oder kann wenigstens sehr leicht so dargestellt werden. Sie umfaßt nicht nur eine enorme Zeitspanne, sondern auch eine enorme Bandbreite der Erfindungen, die von einfachsten Gegenständen wie einer Zeichenfeder bis hin zu den allerkompliziertesten reicht. Von daher verbietet sich jeder Versuch, eine vollständige Geschichtsschreibung der Technik präsentieren zu wollen. Eine Auswahl ist unabdingbar. Glücklicherweise gibt es zwei sehr vernünftige Leitlinien: Wie bedeutsam war eine bestimmte Erfindung oder Serie von Erfindungen?, und in welchem Ausmaß hat sie andere Erfindungen oder Neuerungen stimuliert? Diese beiden Kriterien fallen oft, aber keineswegs immer, zusammen. Es ist durchaus möglich, daß eine Erfindung zwar sehr bedeutsam war, aber keine weiteren Erfindungen angeregt hat.

$\mathrm{Zu}$ den Merkmalen-der Technologie, die mit der Zeit immer mehr an Bedeutung gewonnen haben, gehört die enge Wechselbeziehung zur Wissenschaft. Daraus ergibt sich eine weitere Leitlinie. Diese Wechselbeziehung ist und war schon immer symmetrisch. Die Wissenschaft hat von der Technologie nicht weniger profitiert als umgekehrt. Ungeachtet dessen scheinen sich die Wissenschaftshistoriker und die Technologiehistoriker in zwei völlig getrennte Gruppen aufzuspalten, obwohl ohne gebührende Beachtung der jeweils anderen Seite weder eine befriedigende Wissenschaftsgeschichte noch eine befriedigende Technologiegeschichte geschrieben werden kann. Es ist meine Hoffnung, daß die folgende Arbeit ein Stück weit zum Verständnis dieser offenkundigen Tatsache beiträgt. Es gibt einige Wissenschaftshistoriker, die verächtlich auf die Technologiegeschichte herabgesehen haben; so hat z.B. ein verstorbener Wissenschaftshistoriker den Unterschied zwischen der Wissenschafts- und der Technologiegeschichte als den Unterschied zwischen den Denkern und den Flickschustern beschrieben. In Wirklichkeit verdankt die Wissenschaft der Technologie sehr viel, und eine Wissenschaftsgeschichte ohne Berücksichtigung der Technologie gibt einfach keinen Sinn.

Das vorliegende Buch beruht auf Vorlesungen und Seminaren, die während der vergangenen 25 Jahre am Institut für Wissenschaft und Technologie der Universität von Manchester stattfanden. Ich hoffe, daß es in anderen Instituten für ähnliche Zwecke von Nutzen sein kann. Es liegt in der Natur der Sache, daß es ein Zwischenprodukt darstellt, da es sich mit einem in gewisser Hinsicht neuen Thema befaßt, nämlich mit einer Wissenschaftsund Technologiegeschichte. Deshalb möge es einfach als ein Beitrag gesehen werden, eine integrierte und verwertbare Technologiegeschichte zu schaffen. Die wesentliche Grenze dieses Buches liegt darin, daß es sich hauptsächlich auf physikalische Technologien beschränkt. 
Ich meine, daß es wenigstens einen weiteren, genauso umfangreichen Band erfordern würde, wollte man den Biotechnologien nur einigermaßen Gerechtigkeit widerfahren lassen. Ebenso spielt auch die chemische Technologie nur eine untergeordnete Rolle; der Grund dafür ist, daß bereits exzellente Geschichtswerke über Chemie und Chemietechnologie erhältlich sind. Eine andere Grenze ist, daß man der Meinung sein kann, es betone in unbilliger Weise die englischen Erfahrungen. Ich möchte dem verständnisheischend entgegenhalten, daß die große, als industrielle Revolution bekannte Bewegung unbestreitbar im 18. Jahrhundert von England ihren Ausgang genommen hat, und daß es unrealistisch wäre, von einem Angehörigen des Kernlands dieser Revolution zu erwarten, daß er darüber schreibt, als wäre all dies am anderen Ende der Welt geschehen.

Nun bleibt mir nur noch, meinen Freunden sowie meinen ehemaligen und gegenwärtigen Kollegen für viele anregende Diskussionen, Kommentare und konstruktive Kritik zu danken. Besonders dankbar bin ich Michael Bailey, Dr. K.R. Barlow, Dr. Michael Duffy, Dr. Kathleen Farrar und ihrem Ehegatten, dem verstorbenen Dr. Wilfred Farrar, R.S. Fitzgerald, Dr. Patrick Greene und dem gesamten Personal des Museums der Wissenschaft und der Industrie in Manchester, Rev. Dr. Richard Hills, J.O. Marsh, Dr. Arnold Pacey, Dr. John Pickstone und Dr. Alan Williams sowie allen anderen Mitgliedern der „Manchester-Schule“, den Bibliothekaren und ihren Mitarbeitern in Manchester und an allen anderen Orten, und schließlich, last but not least, vielen Doktoranden und Studenten. Die Verantwortung für eventuelle Fehler kommt selbstverständlich mir allein zu. 


\section{Inhaltsverzeichnis}

1 Einleitung 1

2 Griechische Getriebe $\quad 12$

$3 \quad$ Neue Welten und die Informationsrevolution 31

4 Die wissenschaftliche Revolution $\quad 49$

$\begin{array}{lll}5 & \text { Vernunft und Verbesserung } & 67\end{array}$

$\begin{array}{llr}6 & \text { Verwirklichter Fortschritt } & 83\end{array}$

7 Die Geburt der Fabrik 99

8 Autonome Technologie: Die Eigendynamik des Fortschritts 116

9 Das napoleonische Europa $\quad 132$

10 Straßen, Schienen und eine neue Energiewirtschaft $\quad 148$

11 Die öffentliche Seite der Technologie: Kunstfertigkeit und Intelligenz 164

12 Die Fortschrittsflut $\quad 179$

13 Drei innovative Jahrzehnte 195

14 Eine zweite industrielle Revolution $\quad 214$

15 Das Jahrhundert der Kriege $\quad 234$

$\begin{array}{ll}16 \text { Fallbeispiele } & 253\end{array}$

17 Großtechnologie - die Zukunft im Visier 273

18 Technologie und der Einzelne: Kleine Technologie 294

19 Anmerkungen zu einer Philosophie der Technologie 313

$\begin{array}{ll}\text { Sachwortverzeichnis } & 333\end{array}$ 